\title{
Schweigen zur Initiative «Schutz der Bevölkerung vor Passivrauch»
}

\section{Rainer M. Kaelin}

Dr. med., Facharzt für Pneumologie und Innere Medizin, Morges, vormals Vizepräsident der Lungenliga Schweiz und der Lungenliga Waadt

\section{Einleitung und Hintergrund}

Lange vor der Annahme des Alibi-Bundesgesetzes von 2008 zum Passivrauchschutz hatte die Lungenliga Schweiz vermutet, dass eine Volksabstimmung nötig sein würde, um eine wirksame Gesetzgebung zu erreichen. Diese Strategie wurde durch den Entscheid ihres Delegiertenrates schon im Jahr 2005 bestätigt ein Beschluss, der jährliche finanzielle Rückstellungen der kantonalen Ligen zur Folge hatte. Einige Ligen unterstützten die Volksinitiative "Schutz der Bevölkerung vor Passivrauch" dennoch nicht: Weder die Lungenliga Waadt noch die Lungenliga Zürich positionierten sich öffentlich, auch beteiligten sie sich nicht an der Unterschriftensammlung. Die Lungenliga Waadt positionierte sich erst im Jahr 2010, kurz vor Abschluss der Initiative. Noch ein Jahr zuvor hatte ihre Präsidentin die Lancierung der Volksinitiative in einem offiziellen Brief an den Präsidenten der Lungenliga Schweiz verurteilt [1]*. Diese Haltung stand im Gegensatz zur öffentlichen Meinung, die teilweise durch eine Volksabstimmung bereits vor $2008 \mathrm{zu}$ einer wirksameren kantonalen Gesetzgebung geführt hatte (TI, VS, VD, SO usw.). Gemäss einer Meinungsumfrage im Sommer 2010 standen 60\% der Befragten der Initiative wohlwollend gegenüber. Auf ärztlicher Ebene sprachen alle Argumente für ihre Annahme. Bei der Lancierung der Volksinitiative «Schutz der Bevölkerung vor Passivrauch» bildete die Lungenliga Schweiz eine breite Allianz von Gesundheitsorganisationen, was für erweiterte Sichtbarkeit des Themas und breite Unterstützung bei der Abstimmung sorgen sollte. Regelmässig stellte die Lungenliga Schweiz den Medien und Allianzpartnern Informationen zur Verfügung. Den Organisationen der Allianz standen interne Kommunikationsmittel zur Verfügung, um ihr Zielpublikum zu sensibilisieren - Mitglieder, Angestellte, Spender und Sympathisanten der Lungenliga und der Krebsliga. Es wurde jedoch schon lange vor der Abstimmung klar, dass die Allianz nicht effizient zusammenarbeitete und dass sie ihre eigenen Kommunikationsmittel ungenutzt liess, während die Bevölkerung in Treu und Glauben auf ihren Einsatz vertraute.

\section{Das Schweigen der FMH}

Die Dachorganisation der Ärzteschaft argumentierte noch am 1.4.2009, dass die «Initiative Kräften binden [könnte], [...] die im Kampfe um das neue Präventionsgesetz gebraucht würden». Erst durch einen Beschluss ihrer Delegierten Ende 2009 unterstützte die FMH offiziell die Passivrauchinitiative. Dieser Positionierung folgte keine regelmässige Information ihrer Mitglieder. Bis zur Abstimmungskampagne finden sich im Organ der FMH, der Schweizerischen Ärztezeitung (SÄZ), keine Informationen oder Aufrufe zur Passivrauchschutzinitiative, die von der FMH oder ihrer Abteilung für Prävention gezeichnet wären. Bezeichnend ist die von der SÄZ und der Berner Ärztegesellschaft organisierte öffentliche Podiumsdiskussion «Prävention zwischen sinnvoller Steuerung und Bevormundung» am 6. Juli 2011. Weder in der Ankündigung noch während der Veranstaltung noch in der Berichterstattung darüber in der SÄZ [2] wurde dem Publikum bzw. der Leserschaft erklärt, dass der am Gespräch teilnehmende Hans Ulrich Bigler zwei Hüte trägt: Als Direktor des Schweizerischen Gewerbeverbandes vertritt er die KMUs, er ist aber gleichzeitig Schriftführer der "Allianz der Wirtschaft für eine massvolle Präventionspolitik» [3]. Dieser Zusammenhang ist bedeutsam, denn es ist nicht allgemein bekannt, dass sich hinter der Allianz nicht nur die mit Philip Morris verbundene Gastrosuisse, sondern auch die in der Schweiz ansässigen internationalen Tabakfirmen und die politischen Parteien CVP und SVP als unterstützende Organisationen verbergen. Zu den Gründungsmitgliedern der Allianz gehört EconomieSuisse, die in einer offiziellen Stellungnahme bei der Australischen Regierung die Interessen der Tabakmultis vertrat, indem sie sich gegen neutrale Zigarettenpackungen als Public-Health-Massnahme aussprach [4]. Ihr Vizepräsident war der damalige CEO von Novartis Daniel Vasella.

\section{Das Schweigen SGIM}

Während der Parlamentsdebatte um das Passivrauchschutzgesetz im Jahre 2008 erklärte die Präsi- 
dentin der Schweizerischen Gesellschaft für Innere Medizin (SGIM), nachdem sie brieflich zu einer Positionierung aufgefordert worden war: «Wir haben die Jahresversammlung nicht als rauchfreie Zone deklariert. Ein Grosskongress kann nicht in letzter Minute einen derartigen Schritt organisieren. Im Vorfeld müssen die entsprechenden Mitorganisatoren, die Sponsoren etc. auf die Neuerung aufmerksam gemacht werden" [5]. Indirekt hatte sie dadurch die Positionierung in der Passivrauchdebatte umgangen.

\section{Der Mangel an Transparenz zerstört Vertrauen.}

Die Antwort wirft Licht auf den Druck, dem der Vorstand der SGIM ausgesetzt war [6]. Nach Vorstössen von Mitgliedern beschloss ihre Generalversammlung jedoch im Jahr 2010, die Initiative zu unterstützen. Merkwürdigerweise wurde über das Traktandum ohne Empfehlung des Vorstandes abgestimmt. Entgegen diesem Entscheid der Mitgliedschaft hat sich die SGIM in der Folge aber nie gegenüber ihren Mitgliedern und der Öffentlichkeit zur Passivrauchinitiative oder zur Tabakprävention allgemein geäussert. Dies lässt vermuten, dass auf die Verantwortlichen der SGIM weiterhin Druck ausgeübt wurde.

\section{Das Schweigen der Organisationen der öffentlichen Gesundheit}

Auf die Aufforderung an die Organisatoren des Jahreskongresses «Swiss Public Health" 2012, im Hinblick auf die kommende Volksabstimmung eine Pressekonferenz zu organisieren, wurde geantwortet, das Programm sei bereits abgeschlossen. Die Präsidentin präzisierte darauf in einer Mail vom 12.9.2012 an den Anfragenden, dass «[...] sich Public Health Schweiz [...] neben dem Präventionsgesetz nicht noch für einen zweiten Gesetzesvorschlag mit gleicher Kraft engagieren kann». Das Schweigen der Experten der öffentlichen Gesundheit wird noch offensichtlicher in der Ausgabe der Revue Médicale Suisse vom 11.7.2012, also im Sommer vor der Abstimmung. Das ganze Heft handelt von Prävention, das Editorial und ein dem Thema gewidmeter Artikel gehen auf die strukturelle Tabakprävention ein, aber nirgends findet sich ein Wort zur Volksbefragung der Passivrauchinitiative im Herbst. Einer der Autoren des Editorials erklärte denn auch anlässlich der öffentlichen Manifestation «Schutz gegen den Passivrauch" einige Wochen vor der Abstimmung, dass er sich in der öffentlichen Debatte um die Passivrauchinitiative nicht positionieren könne, weil sein Institut von öffentlichen Geldern abhängig sei [7].

\section{Das Schweigen der Arbeitsgemeinschaft Tabakprävention (AT)}

Diese Koordinationsorganisation, Mitglied der Gesundheitsallianz, war auch im Initiativkommittee «Schutz der Bevölkerung vor Passivrauch» vertreten. Als im März 2012 nach den Debatten in Bundesrat und Parlament die Bundeskanzlei anfragte, ob die Initiative aufrechterhalten werde, plädierte die Vertreterin der AT überraschend für deren Rückzug. Die Argumentation ihres Komitees unterstützte dieses Votum [8]. Als Motiv wurde die befürchtete Abstimmungsniederlage genannt, der Rückzug hingegen als «staatspolitisch vernünftiger Akt» eingestuft, der «weiteren Anliegen Goodwill verschafft» [...]. «Festhalten an der Initiative wird als Zwängerei interpretiert werden und kann das Scheitern des Präventionsgesetzes bedeuten.» Der Hinweis auf das Präventionsgesetz findet sich auch in den Weigerungen der FMH und Swiss Public Health, sich zu positionieren (s. oben). Die Verbindung der beiden Präventionsprojekte könnte auf eine heimliche Absprache hinweisen, etwa nach dem Motto: Falls die Passivrauchinitiative fallengelassen wird, lassen wir das Präventionsgesetz im Parlament durchkommen. Für diese Interpretation spricht, dass die Tabakindustrie eine analoge Strategie schon einmal in Kalifornien angewendet hatte. Ihre Kommunikationsexperten hatten damals geraten, darauf hinzuarbeiten, eine Abstimmung zu verhindern; diese Strategie wäre für die Tabakindustrie selbst dann nützlich, wenn sie letztlich misslingen sollte. Denn die zu orchestrierende Desorganisation der Abstimmungsbefürworter bedeutete für die Industrie einen Vorsprung in der Abstimmungskampagne und würde in jedem Fall allen Tabakgegnern vor Augen führen, wie mühsam es sein kann, sich mit ihr anzulegen. Die Kommunikationsexperten setzten als erstes Teilziel dieser Strategie, die kalifornische Ärztegesellschaft zu behindern [9].

\section{Lehren aus dem Expertenschweigen}

Die beschriebenen Ereignisse allein dem Zufall oder Kommunikationsfehlern unerfahrener Vertreter von Ärzte- und Gesundheitsorganisationen zuzuschreiben, ist nicht plausibel. Im Ganzen betrachtet, erscheinen sie als ein Verhalten, das klar den Präventionsbemühungen schadet und das man einem Einflussnetzwerk zuordnen kann, so wie man es im Medikamentenmarkt beobachtet (Teil 1, SÄZ 18/2015). Das Prinzip von Treu und Glauben wurde auf mehreren Ebenen verletzt. Das Expertenschweigen widerspricht dem Verhalten, das sich die Organisationen selbst als Prinzi- 
pien vorgeben und das von ihren eigenen Mitgliedern erwartet wird. Alle genannten Organisationen nennen in ihren Statuten die Information der Öffentlichkeit und die öffentliche Gesundheit als ihre Anliegen. Beispiele: "Gesundheitsförderung und Prävention spielen eine immer grössere Rolle im Gesundheitssystem und sind ein erstrangiges Aktionsfeld, auch für die Ärzte: als Experten für die Inhalte der Prävention und der Gesundheitsförderung gegenüber der Öffentlichkeit [...]» (FMH-Website). «Public Health Schweiz will Prävention und Gesundheitsförderung stärken. Durch unsere Aktionen treten wir für dieses Ziel ein und stehen damit immer im Zentrum der Aktualität» (Website von Swiss Public Health). Oder: «[Ziele]: die Öffentlichkeit und die Medien über Themen der Gesundheit und der Gesundheitspolitik informieren [...]" (Statuten Société vaudoise de médecine, 2008).

Das Schweigen der ärztlichen Experten widerspricht Empfehlungen internationaler Organisationen, von denen die Öffentlichkeit den Einsatz für die Tabakprävention erwartet. Die WHO-Rahmenkonvention [10] hält fest: "Strong political commitment is necessary [...] The participation of civil society is essential in achieving the objective of the Convention [...]». Und Ärztegesellschaften werden aufgerufen, sich einzusetzen: "[...] In addition to their role as citizens, physicians are leaders with regard to any issues affecting public health. Physicians should therefore be aware that public policy regarding smoking can be an effective instrument of public health, and they have the responsibility to participate effectively in public debate both as individuals and as members of medical organizations" [11].

Ärzte werden von Patienten und Gesellschaft als vertrauenswürdige, von Pressionen unabhängige Experten angesehen. Seit es Ärzte gibt, werden sie in Fragen gesunder Lebensführung konsultiert. Dass Fragen der öffentlichen Gesundheitspolitik nicht in ihre Kompetenz fielen, weil sie den Rahmen der individuellen Arzt-Patienten-Beziehung sprengen, kann als Argument nicht akzeptiert werden. Denn einerseits haben zahlreiche individuelle ärztliche Massnahmen zwangsläufig Folgen auf das Umfeld der Patienten, z.B. die Behandlung von Infektionskrankheiten, die Isolierung kranker Patienten usw. Hier wird als selbstverständlich angenommen, dass Ärzte sowohl im Interesse der Patienten als auch der Öffentlichkeit agieren. Andererseits nehmen Ärzteorganisationen seit jeher an der politischen Debatte teil mit der legitimen Absicht mitzugestalten («keine Gesundheitspolitik ohne die FMH»). Die Öffentlichkeit erwartet mit Recht, dass Ärzte in Fragen der Prävention einen Standpunkt vertreten, den man als «Gesundheitsstandpunkt» bezeichnen kann und der unabhängig ist von Meinungen, die als Ausdruck von Partikularinteressen verdächtigt werden müssen.

Das Schweigen der ärztlichen Experten wiederholt sich zurzeit in der öffentlichen Diskussion über den bundesrätlichen Entwurf zum Tabakproduktegesetz. In einer gemeinsamen Stellungnahme vom 27.6.2014 [12] zum Entwurf des Tabakproduktegesetzes des Bundesrates [13] äussern sich die Krebsliga, die Lungenliga, die schweizerische Herzstiftung und die Abeitsgemeinschaft Tabakprävention der Schweiz. Es fehlen jedoch die Ärzte: Weder die FMH noch Swiss Public Health haben sich bisher öffentlich zur Vernehmlassung hören lassen. Dabei ist offensichtlich, dass die Präventionsgegner keine Gelegenheit und kein noch so schwaches Argument ungenützt lassen werden, um vom Parlament ein der Industrie möglichst genehmes Gesetz verabschieden zu lassen. Unter den Argumenten, die in der Parlamentsdebatte zu erwarten sind, wird das Schweigen der Ärzteschaft schwer wiegen, da dieses mit Sicherheit als Unterstützung einer «moderaten» Lösung interpretiert werden wird, im Gegensatz zur «Extremlösung» der Präventionsfachleute, eine wohlerprobte Methode, diese als Eiferer zu verunglimpfen.

\section{Schlussfolgerung}

Die jüngste Vergangenheit hat erneut gezeigt, dass staatliche Behörden und Parlamentarier dem enormen Druck der Wirtschaftslobby (14-16) ausgesetzt sind, die sich gegen jede Transparenz stemmt $[17,18]$ Der Mangel an Transparenz zerstört das Vertrauen der Gesellschaft gegenüber privaten und öffentlichen Institutionen. Dies spiegelt sich auch im wachsenden Misstrauen gegen multinationale Firmen, Banken und die Pharmaindustrie (19-21). Im Gewirr von Interessenkonflikten, welche die selbst unter Druck stehenden Medien nur ungenügend beleuchten, darf man von Ärzteorganisationen erwarten, dass sie ihre Expertenrolle im Interesse der öffentlichen Gesundheit in Treu und Glauben wahrnehmen.

In der heutigen von Wirtschaftsinteressen dominierten Welt, in der Information, Desinformation und politische Meinungen analog zur Vermarktung von Konsumgütern aller Art von globalen Akteuren ohne Transparenz durch die Arbeit von PR-Spezialisten organisiert werden, zerstört das Schweigen der ärztlichen Experten das gesellschaftliche Klima von Treu und Glauben. Dies schadet auch den demokratischen Institutionen, die nur in einem Umfeld funktionieren können, in dem die Entscheidungsträger Zugang zu einer umfassenden, seriösen und von Partikularinteressen unabhängigen Information haben. 
1 G.N. Brief der Präsidentin der Lungenliga Waadt an den Präsidenten der Lungenliga Schweiz O.Piller, 23.12.2008.

2 Witte F. Prävention ist geteilte Verantwortung. Schweiz Ärztezeitung. 2011;92(34):1276-9.

3 Die Allianz der Wirtschaft für eine massvolle Präventionspolitik umfasst folgende Organisationen: Schweizerischer Gewerbeverband, ASCO (Verein der Konzertlokale, Cabarets, Dancings und Discotheken), GastroSuisse, HotellerieSuisse, Schweizerischer Bauernverband, Union der Casinos, Schweizer Werbung, Schweizer Zigarrenfabrikanten und Tabakhändler, VISCOM (Verein für visuelle Kommunikation ) etc. Unter den unterstützenden Organisationen finden sich die christliche Volkspartei (CVP), die Schweizerische Volkspartei und Swiss Cigarette (Philip Morris, British American Tobacco, Japan Tobacco International)

4 Thomas Pletscher, EconomieSuisse: Consultation on the exposure draft Tobacco plain packaging Bill 2011 and Consultation Paper to Department of Health and Ageing. Canberra, Australia. May 192011

5 V.B. Präsidentin der SGIM. Brief vom 3.4.2008

6 Dass die Tabakindustrie interveniert hat, erscheint wenig wahrscheinlich. Es ist jedoch nicht ausgeschlossen, dass die Pharmaindustrie, seit Jahrzehnten wichtigster Sponsor der Fortbildungsveranstaltungen der Ärztekongresse und wichtiger Geldgeber der klinischen Forschung eine Rolle gespielt hat.

7 F.P. Institut universitaire de médecine sociale et préventive; Informationsveranstaltung vom 29.8.2012 im Hinblick auf die Volksabstimmung Schutz der Bevölkerung vor Passivrauch» vom 23. September 2012.

8 Komitee der AT, Bern, 20.3.2012: «Ja zum Rückzug der Initiative «Schutz der Bevölkerung vor Passivrauch». An die Mitglieder des Initiativkommittees. ... Mit einem klaren Mehrheitsbeschluss empfiehlt das Komitee der AT, die Initiative zurückzuziehen ... die wichtigsten Organisationen der Prävention, welche im Komitee der AT vertreten sind, haben ausgedrückt, dass sie den Rückzug empfehlen, dass sie dies auch so kommunizieren werden und entsprechend auch die Abstimmungskampagne nicht unterstützen werden (4 Seiten, gezeichnet B.M. Präsident).

9 Paul Kinney, A-K Associates. To Roger Mozingo and Hurst Marshall. Personal and confidential. Sept 24.1987. (http://legacy. library.ucsf.edu./tid/diz44d00/pdf 506609215.page3. The tobacco industry prudently recognized that effort and resources spent in keeping the proposed tax initiative off the ballot had many benefits. If successful, it would save the industry the many millions that would have to go into the initiative campaign fight. Even if unsuccessful, the relative small amount of resources expended on the organizational efforts to keep the initiative off the ballot, would place the industry miles ahead for the general campaign. This unique and innovative approach of actively fighting to keep the initiative off the ballot would only reinforce to the potential anti-tobacco forces that it will always be a long and gruelling battle when you take on tobacco. Recognizing that the most effective approach to any such battle is to contain it and if possible take away potential resources from the proponents, our initial goal was to contain the California Medical Association...

10 www.who.int/tobacco/framework.

11 Joint committee of American college of chest physicians, American thoracic society. Asia Pacific Society of Respirology, Canadian Thoracic Society, European Respiratory Society, International Union against Tuberculosis. In: Eur Respir J. 1995;(8):1808-11.

12 Vernehmlassung zum Vorprojekt zu einem neuen Tabakproduktegesetz (TabPG). Bern 27.6.2014. Korrespondenzadresse Lungenliga Schweiz, Chutzenstrasse Bern.

13 Kaelin RM. Entwurf des Bundesrates zum Tabakproduktegesetz. Unterwanderung des Jugendschutzes und Verletzung der WHO-Rahmenkonvention . Schweiz Ärztezeitung. 95(37):1383-4.

14 Petitjean S. Philip Morris accusé de conspiration à Bruxelles. Le Temps. 5 juillet 2014.

15 Petitjean S. José Manuel Barroso dément toute entente avec l'industrie du tabac. Le Temps, 12 juillet 2014.

16 Das Geschäft mit dem Gift - Die Schweiz ist für Tabakkonzerne ein Traumland. Beobachter. 31. Oktober 2014

17 Wuthrich B. Pas d'accréditation pour les lobbyistes fédéraux. Transparence. Nouvelle règle rejetée. Le Temps. 3 juin 2014.

18 Boder W. Les dons des entreprises aux partis resteront secrets. Le Temps. 11 juin 2014.

19 Boder W. Les pharmas doivent affronter une crise de confiance de leurs clients. Le Temps. 13 mai 2014.

20 Boder W. La populaton déplore le manque de transparence de la pharma. Le Temps, 25 juin 2014.

21 Borch-Jacobsen M (Koordination). Big Pharma. Une industrie toute-puissante, qui joue avec notre santé. Paris: Les Arènes; 2013. Beiträge verschiedener nordamerikanscher und europäischer Autoren. 